\title{
Originales
}

\section{Actitudes y percepciones del personal médico del hospital acerca de los Cuidados Intensivos y de la especialidad de Medicina Intensiva}

\author{
L. SANTANA CABRERA, M. SÁNCHEZ-PALACIOS, F. RODRÍGUEZ GONZÁLEZ, \\ E. HERNÁNDEZ MEDINA, A. CASAMITJANA ORTEGA Y M. FERNÁNDEZ ARROYO
}

Servicio de Medicina Intensiva. Hospital Universitario Insular de Gran Canaria. Las Palmas de Gran Canaria. Canarias. España.

\begin{abstract}
Objetivo. Evaluar las actitudes y percepciones de los médicos de un hospital respecto a la labor asistencial del Servicio de Medicina Intensiva en su hospital y a la especialidad de Medicina Intensiva.

Diseño. Se trata de un estudio prospectivo, descriptivo, de 3 meses de duración.

Ámbito. Hospital Universitario Insular de Gran Canaria, Canarias, España.

Pacientes. Médicos no intensivistas, adjuntos y residentes del hospital.

Intervención. Se llevó a cabo una encuesta anónima.

Variables de interés principales. Se recogieron datos demográficos de los médicos entrevistados, se les preguntó acerca de su relación laboral o personal previa con la Unidad de Cuidados Intensivos (UCl), las actitudes de los médicos intensivistas de su hospital y su percepción de la especialidad de Medicina Intensiva.

Resultados. Se entrevistaron 116 médicos, 75 adjuntos y 41 residentes. Menos de la tercera parte creía que la UCI era una sala para enfermos potencialmente críticos. Un porcentaje elevado de los médicos a los que se les había denegado algún ingreso no estaba conforme con los argumentos que se les dieron. Más del $40 \%$ de los entrevistados afirmó no haber solicitado cama en la UCI para algún paciente que probablemente se
\end{abstract}

Correspondencia: Dr. L. Santana Cabrera.

Servicio de Medicina Intensiva.

Hospital Universitario Insular de Gran Canaria.

Avda. Marítima del Sur, $\mathrm{s} / \mathrm{n}$.

35016 Las Palmas de Gran Canaria. Canarias. España.

Correo electrónico: 1sancabx@gobiernodecanarias.org

Manuscrito aceptado el 9-I-2008. hubiese beneficiado de ella, sobre todo porque pensaba que se le iba a denegar. Todavía hay médicos en el hospital que piensan que los profesionales que trabajan en la $\mathrm{UCI}$ son médicos de distintas especialidades. La especialidad de Medicina Intensiva es percibida con un alto nivel de estrés entre los profesionales.

Conclusiones. Detectamos el desconocimiento de otros profesionales acerca de la función de un Servicio de Medicina Intensiva y del modelo de $\mathrm{UCI}$ de nuestro país. También encontramos unas elevadas tasas de insatisfacción entre los profesionales a los cuales se les denegó algún ingreso en la unidad, por lo que creemos que se deben elaborar unas guías clínicas sobre los criterios de ingreso y darlos a conocer entre los médicos del hospital. Los profesionales perciben que sufrimos un alto nivel de estrés.

PALABRAS CLAVE: Cuidados Intensivos, calidad de cuidados, percepción social, actitudes del personal sanitario.

\section{PHYSICIANS' ATTITUDES AND PERCEPTIONS REGARDING THE CRITICAL CARE AND CRITICAL CARE SPECIALITY}

Objetive. To evaluate the attitudes and perceptions of the doctors of a hospital regarding critical care and the speciality of Intensive Medicine.

Design. Prospective, descriptive study during 3 months.

Setting. Insular University Hospital in Gran Canaria, Canary Islands, Spain.

Patients. Medical specialists and residents.

Intervention. Anonymous survey.

Main variables. Demographic data of the interviewed doctors were collected and they were 
asked about their labour or personal previous relation with the Intensive Care Unit (ICU), the attitudes of the critical care doctors' of their hospital and their perceptions regarding the critical care speciality.

Results. We interviewed 116 doctors, 75 staff and 41 residents. Less of the third part believed that the ICU was a ward for potentially critical patients. A high percentage of the doctors whom had been refused some admission were not to agree with the arguments that they received. More than $40 \%$ of the interviewed ones affirmed had not requested bed in the ICU for any patient who had benefited from it, especially because they were thinking that the admission would be refused. Some doctors think that the professionals employed at the ICU are doctors of different specialities. The speciality of Intensive Medicine is perceived by a high level of stress among the professionals.

Conclusions. We detect the ignorance of other professionals about the function of a Service of Intensive Medicine and the model of ICU of our country. Also we find high rates of dissatisfaction among the professionals who was refused some admission in the unit, because of we believe that clinical guides about the criteria of admission must be elaborated and to announce them among the doctors of the hospital. The professionals perceive that we suffer a high level of stress.

KEY WORDS: intensive care, quality of health care, social perception, attitude of health personnel.

\section{INTRODUCCIÓN}

La Comisión Nacional de Medicina Intensiva define la Medicina Intensiva como aquella que se ocupa de los pacientes con disfunción actual o potencial de uno o varios órganos que representa una amenaza para su vida y son susceptibles de recuperación ${ }^{1}$; del mismo modo se define al paciente crítico como aquel que se halla en riesgo vital inmediato o posible, cuya situación clínica sea reversible, incluyendo el soporte del potencial donante de órganos ${ }^{2}$.

Aunque existen pocos datos en la literatura que validen los criterios de ingreso en la Unidad de Cuidados Intensivos (UCI), sí que existe un consenso llevado a cabo en una reunión de expertos de la American College of Critical Care Medicine, que motivó la publicación de unas guías acerca de los criterios de ingreso, alta y triage de pacientes adultos en $\mathrm{UCI}^{3}$.

La especialidad de Medicina Intensiva en España ha sido y sigue siendo una gran desconocida entre la población general, tal como lo ha demostrado algún trabajo publicado al respecto ${ }^{4}$, pero no sabemos cuál es la percepción de los médicos no intensivistas sobre la especialidad de Medicina Intensiva, cuáles son las características que más les atraen o desagradan y las diferencias con su especialidad; aspectos evaluados en la literatura con escasos trabajos llevados a cabo en otros países que no siguen el modelo español de la especialidad ${ }^{5,6}$.

Por otro lado, cada día se le da más importancia a la calidad de los servicios prestados en nuestros hospitales y, por supuesto, en los Servicios de Medicina Intensiva $^{7-10}$. Con la intención de mejorar la atención del paciente crítico, la Sociedad Española de Medicina Intensiva Crítica y Unidades Coronarias (SEMICYUC) elaboró el libro titulado Indicadores de calidad en el enfermo crítico $^{11}$, donde se elaboraron 120 indicadores, de los cuales 20 se consideraron relevantes. Trabajando en esta línea de intentar mejorar los servicios, son muchos los estudios llevados a cabo para identificar las necesidades de los familiares o de los pacientes que ingresan en $\mathrm{UCI}^{12}$, aunque también creemos que nuestros propios compañeros médicos no intensivistas nos pueden dar una valiosa información sobre la calidad percibida de los Cuidados Intensivos que estamos prestando y poder así implantar un plan de mejoras.

Por tanto, los objetivos de nuestro estudio eran valorar las actitudes y percepciones de los médicos no intensivistas de nuestro hospital sobre la especialidad de Medicina Intensiva y los Cuidados Intensivos prestados.

\section{PACIENTES Y MÉTODO}

Se trata de un estudio prospectivo, descriptivo, realizado desde abril hasta junio de 2007, en el Hospital Universitario Insular de Gran Canaria, hospital terciario con una capacidad de 425 camas, 450 facultativos y 110 residentes. Este centro posee una UCI con unas instalaciones modernas, inaugurada en el año 2001, con 24 camas polivalentes (médico-quirúrgica y coronaria), una plantilla de 13 facultativos especialistas en Medicina Intensiva y 7 residentes.

El estudio incluía a los profesionales médicos no intensivistas, tanto adjuntos como residentes, pertenecientes a la plantilla de nuestro hospital, que habían trabajado en nuestro medio un periodo superior a los 6 meses y que, por su especialidad, se suponía que podían haber tenido alguna relación laboral con el Servicio de Medicina Intensiva. Se utilizó una encuesta anónima (anexo 1) diseñada por un grupo de facultativos de Medicina Intensiva, la cual evalúa la existencia de una relación previa de los médicos encuestados con la UCI, ya sea laboral o personal, su percepción basada en dicha relación, el conocimiento de los encuestados sobre la especialidad de Medicina Intensiva, sus profesionales y las características de la especialidad que pudieran diferenciarla de otras. Las cuestiones acerca del conocimiento sobre la UCI fueron adaptadas de las publicadas por García-Labattut et $\mathrm{al}^{4}$, mientras que las preguntas acerca de la percepción de la especialidad fueron adaptadas a nuestro entorno de las publicadas por Lorin et $\mathrm{al}^{6}$.

El análisis estadístico se efectuó mediante el paquete estadístico SPSS 10.0, los datos fueron presentados como medias \pm desviación estándar. El cálculo 


\section{ANEXO 1. ENCUESTA DE VALORACIÓN DE LAS ACTITUDES Y PERCEPCIONES DEL PERSONAL MÉDICO DEL HOSPITAL ACERCA DE LOS CUIDADOS INTENSIVOS}

Estimado compañero/a:

La Dirección de nuestro hospital y los médicos del Servicio de Medicina Intensiva estamos interesados en conocer la percepción que tienen los miembros del personal facultativo sobre los Cuidados Intensivos prestados en nuestro hospital, con el propósito de detectar factores de mejora que pueden influir en una mejor atención al usuario.

Por este motivo nos dirigimos a usted, con el fin de solicitar su colaboración en la cumplimentación de esta pequeña encuesta, con la seguridad de que ella nos será de gran utilidad. Su colaboración es totalmente voluntaria y todo lo que diga será tratado de manera confidencial.

1. Edad:

Varón

Hembra

3. Categoría:

Médico residente: especialidad

Facultativos: especialidad

Médica Quirúrgica Serv. Central

Médica Quirúrgica Serv. Central
Año de especialidad

Años de ejercicio

4. ¿Ha realizado alguna rotación de estudiante o residente por una unidad de Medicina Intensiva? (Meses)

5. ¿Ha trabajado en alguna Unidad de Medicina Intensiva? (Meses)

6. ¿Conoce personalmente? (Conteste sí o no)

La ubicación de la Unidad de Cuidados Intensivos (UCI)

Al jefe de la unidad o al supervisor

A algún médico adjunto o residente

A algún otro miembro del personal sanitario

7. ¿Ha estado ingresado usted o algún familiar directo alguna vez en la UCI? (Días)

8. ¿Cómo calificaría usted la experiencia? (Puntúe de $\mathbf{0}=$ terrible a $\mathbf{1 0}=$ perfecto)

9. ¿Ha tenido algún contacto profesional con el servicio de Medicina Intensiva? (Señale con una $X$ las que considere)

Le han solicitado consulta desde la UCI

Ha solicitado usted el ingreso de un paciente en la UCI

Ha trasladado algún paciente a la UCI

Ha recibido algún paciente desde la UCI

Otros (especificar)

No he tenido ningún contacto

10. ¿Qué es para usted una UCI? (Señale con una X las que considere acertadas)

Una sala para enfermos con respirador

Una sala donde se controla las 24 horas del día el ritmo del corazón

Una sala para enfermos terminales irrecuperables y que se ingresan para evitarles el sufrimiento

Una sala para enfermos que aunque no estén críticos pudiesen estarlo en cualquier momento

Una sala para el tratamiento de pacientes recuperables con una enfermedad que se encuentre en una fase crítica

Una sala que pueda tratar enfermos que, aun no estando en fase crítica, supongan una sobrecarga

de trabajo para una planta de hospitalización

11. ¿Se le ha denegado el ingreso de algún paciente en la unidad que usted creía que era subsidiario de tratamiento intensivo? (Señale con una $\mathrm{X}$ el que considere)
Sí
No

12. En caso afirmativo, ¿se quedó usted conforme con los argumentos que se le dieron para no ingresarlo? (Señale con una $X$ el que considere)
Sí
No

13. Si ha contestado No a la pregunta anterior: ¿cuáles fueron los motivos? (Señálelos) 
ANEXO 1. ENCUESTA DE VALORACIÓN DE LAS ACTITUDES Y PERCEPCIONES DEL PERSONAL MÉDICO DEL HOSPITAL ACERCA DE LOS CUIDADOS INTENSIVOS (Continuación)

14. Si en algún momento no ha solicitado la cama en la UCI para un paciente que creía que se hubiese beneficiado de ella, ¿cuál fue la causa? (Señale las que considere correctas)

Dar por hecho que no hay camas disponibles en la UCI

Pensar que se le va a denegar el ingreso en la UCI

No tener claros los criterios de ingreso en la UCI

Pensar que se podía tratar de igual forma en planta

Otras: especificar

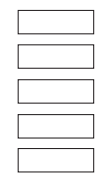

15. ¿Cuáles cree usted que son razones justificadas para no ingresar a un paciente en la UCI? (Conteste sí o no)

La edad .

La situación basal

El pronóstico a corto plazo

Las secuelas que le pueden quedar

Negativa del paciente o de la familia

La última cama libre en la UCI si el estado del paciente lo permite

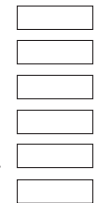

16. ¿Qué titulación tienen los médicos de la UCI? (Señale con una X)

Son de distintas especialidades

No son especialistas, sino médicos generales

Son médicos con título específico de especialistas en Medicina Intensiva ...........

17. ¿Qué percepción tiene de la especialidad de Medicina Intensiva comparada con la suya? (1: mucho menos; 2: algo menos; 3: similar; 4: algo más; 5: mucho más)

Nivel de estrés

Horas de trabajo ....

Influencia en la vida personal

Prestigio dentro de la profesión médica

Prestigio dentro de la institución

Prestigio a nivel social

Riesgo de mala práctica

Disponibilidad laboral

Sensación de satisfacción del personal

18. Elegir las 3 características más atractivas de la Medicina Intensiva (Asignar el orden $1 .^{\circ}, 2^{\circ}$ y $3^{\circ}$ )

Estímulo intelectual

Tratar enfermos en situación crítica

Poder aplicar conceptos fisiológicos complejos

La realización de procedimientos y técnicas .....

El contacto con cuestiones del final de la vida

La oportunidad de coordinar varios servicios en el tratamiento de un paciente ...

El contacto con cuestiones éticas complejas

Estar de acuerdo con mi personalidad

Tratar enfermos de forma crónica ....

Tener tiempo de ocio durante el trabajo

Buen ambiente y relax entre el personal

19. Elegir las 3 características menos atractivas de Medicina Intensiva (Asignar el orden $1 .^{\circ}, 2^{\circ}$ y $3 .^{\circ}$ )

Falta de estímulo intelectual

Tratar enfermos en situación crítica ....

Tener que aplicar conceptos fisiológicos complejos

La realización de procedimientos y técnicas que se llevan a cabo

El contacto con cuestiones del final de la vida

Faltan oportunidades para coordinar varios servicios en el tratamiento

El contacto con cuestiones éticas complejas

El estrés entre el personal

Ser incompatible con mi personalidad

Tratar enfermos de forma crónica

Falta de tiempo libre, demanda demasiado tiempo y esfuerzo

Mal ambiente entre el personal

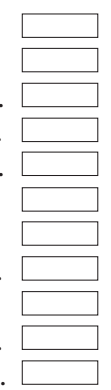


TABLA 1. Características de los profesionales entrevistados

\begin{tabular}{|l|c|c|}
\hline & $\begin{array}{c}\text { Adjuntos } \\
(\mathrm{n}=75)\end{array}$ & $\begin{array}{c}\text { Residentes } \\
(\mathrm{n}=41)\end{array}$ \\
\hline Edad & $41,8 \pm 8,5$ & $28,2 \pm 4,4$ \\
Sexo & & \\
$\quad$ Varones & $53(70,6 \%)$ & $18(43,9 \%)$ \\
Mujeres & $22(29,3 \%)$ & $23(56,1 \%)$ \\
Especialidad, $n$ & & 21 \\
Médica & 39 & 4 \\
Quirúrgica & 14 & 7 \\
Servicios Centrales & 12 & 9 \\
Urgencias & 10 & $2,17 \pm 1,1$ \\
Tiempo trabajado en el hospital (años) & $12,9 \pm 9,4$ & $17(41,4 \%)$ \\
Rotaron por la UCI, n (\%) & $39(52 \%)$ & $1,9 \pm 1,1$ \\
Duración (meses) & $3,8 \pm 2,3$ & 0 \\
Trabajaron en alguna UCI, n (\%) & $12(16 \%)$ & $\mathrm{n}=41$ \\
Duración (meses) & $43 \pm 62,2$ & $37(90,2 \%)$ \\
Conocimiento personal de la UCI & $\mathrm{n}=75$ & $18(43,9 \%)$ \\
Ubicación & $74(98,6 \%)$ & $39(95,1 \%)$ \\
Jefe de la Unidad o supervisor & $65(86,6 \%)$ & $29(70,7 \%)$ \\
Médicos & $74(98,6 \%)$ & $\mathrm{n}=38$ \\
Otro personal sanitario & $65(86,6 \%)$ & $19(50 \%)$ \\
Contacto profesional con la UCI, & $\mathrm{n}=75$ & $21(55,2 \%)$ \\
Consulta desde la UCI & $65(86,6 \%)$ & $22(57,9 \%)$ \\
Ingreso en UCI & $56(74,6 \%)$ & $28(73,7 \%)$ \\
Traslados a la UCI & $53(70,6 \%)$ & $1(2,6 \%)$ \\
Traslados desde la UCI & $53(70,6 \%)$ & $3(7,9 \%)$ \\
Otros & $1(1,3 \%)$ & \\
Ningún contacto & 0 & \\
\hline
\end{tabular}

UCI: Unidad de Cuidados Intensivos.

de la muestra se basa en que para una población de 350 médicos, una confianza del $95 \%$ y una precisión del 7\%, el tamaño de muestra a utilizar es de 116 encuestas.

\section{RESULTADOS}

Se distribuyeron 350 encuestas entre los médicos adjuntos y residentes del hospital y se devolvieron cumplimentadas $116(33,1 \%)$, de las cuales $75(64,6 \%)$ correspondían a médicos adjuntos y $41(36,4 \%)$ a médicos residentes en formación. Las características demográficas de los profesionales entrevistados se encuentran en la tabla 1, donde destaca que los médicos adjuntos tenían una experiencia profesional superior a los 10 años y todos habían tenido algún contacto personal o profesional con la UCI; de hecho, el 16\% de los adjuntos entrevistados había trabajado en alguna UCI (pública o privada) en algún momento de su trayectoria profesional. En cuanto a los médicos residentes, todos conocían personalmente a algún miembro del personal que trabajaba en la Unidad y el $92,1 \%$ había tenido algún contacto profesional.

Dieciocho (24\%) de los adjuntos y $8(19,5 \%)$ de los residentes habían tenido algún familiar directo ingresado en la UCI, por lo que se les pidió que evaluaran de 0 (terrible) a 10 (perfecto) la experiencia vivida en la UCI. El 77,7\% de los adjuntos y el 62,5\% de los residentes le dieron una puntuación de 6 puntos o superior (fig. 1).

También se les preguntó qué era para ellos una UCI y, mientras casi todos los adjuntos y residentes estaban de acuerdo en que era una sala para pacientes recuperables en fase crítica, sólo el $29 \%$ de los adjuntos y el $24 \%$ de los residentes eligieron la opción de que era una sala para enfermos potencialmente críticos (tabla 2).

Al $64,7 \%$ de los adjuntos y al 48,7\% de los residentes encuestados se les había denegado en alguna ocasión la solicitud de ingreso de algún paciente en la UCI. De ellos, sólo el 21,7 y el 35\%, respectivamente, estaban conformes con los argumentos que se les dieron para no aceptar el ingreso. Los motivos de la disconformidad, para los médicos adjuntos, fueron en la mayoría de los casos la disparidad de criterios en cuanto al beneficio de tratamiento en la UCI en la situación en que se encontraba el paciente $(48,2 \%)$, a los que le seguía el pronóstico a corto plazo $(40,3 \%)$, la última cama $(6,5 \%)$ y la edad y las secuelas que les podían quedar $(2,5 \%)$. Para los residentes también los motivos principales que se les esgrimieron para no admitir el ingreso fue, en el 49,5\%, la diferente percepción con el intensivista en cuanto a la situación en que se encontraba el paciente (fig. 2).

Treinta y tres (44\%) de los médicos adjuntos y 18 (44\%) de los residentes encuestados afirmaron no haber solicitado, al menos en alguna ocasión, cama en la UCI para un paciente que creían que se hubiese beneficiado de ella, y la causa más frecuente para ambos grupos fue «pensar que se le va a denegar el ingreso en la UCI». El 15\% de los adjuntos y el $22 \%$ de los residentes no tenían claros los criterios de ingreso en la UCI (tabla 3 ).

También se les preguntó cuáles eran para ellos razones justificadas para no ingresar a un paciente en la UCI, a lo que un $77 \%$ de los adjuntos respondió la 


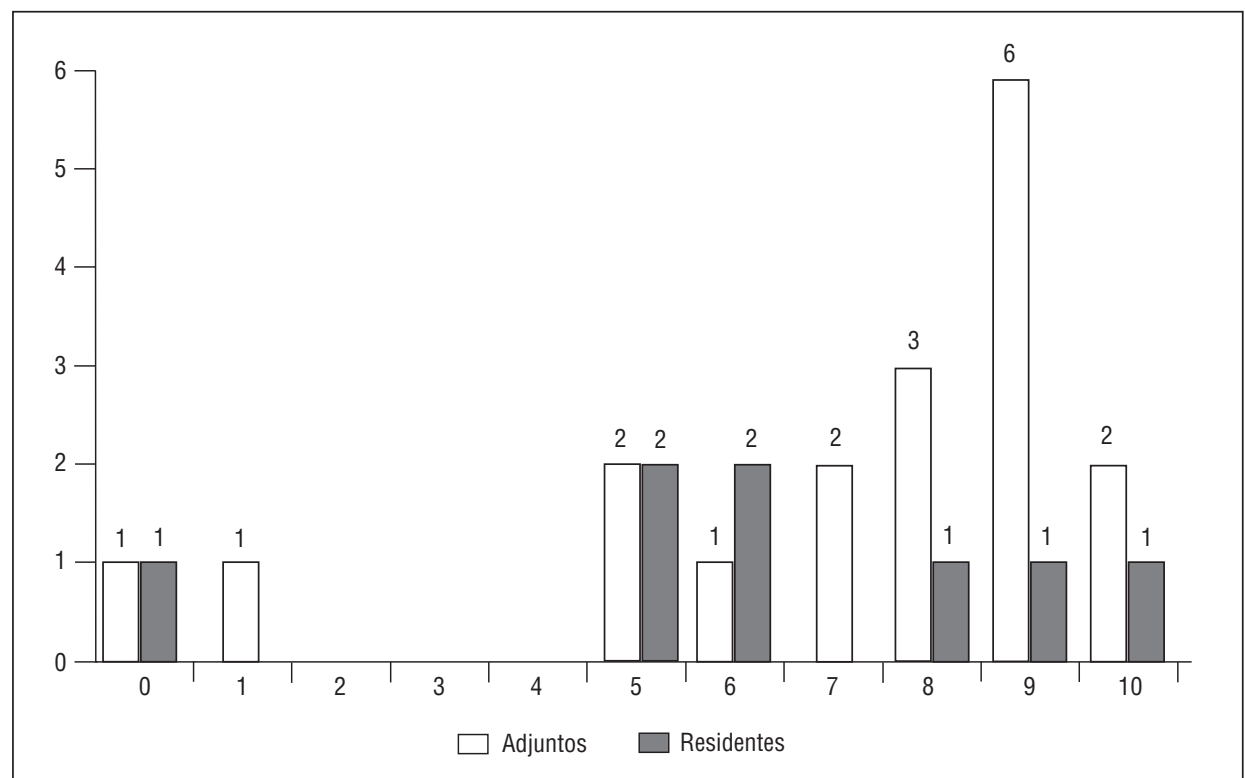

Figura 1. Valoración de la experiencia vivida en la Unidad de Cuidados Intensivos por los profesionales que tuvieron algún familiar ingresado en ella. Calificación de la experiencia vivida: 0: terrible, 10: perfecto.

«negativa del paciente o la familia» y el $79 \%$ de los residentes: «la situación basal» del paciente (tabla 4).

En la encuesta se incluyó una pregunta sobre la titulación de los médicos de la UCI y el $4 \%$ de los adjuntos aún pensaba que eran médicos de distintas especialidades y el $1,3 \%$ que no eran especialistas, sino médicos generales.

En cuanto a la percepción de la especialidad de Medicina Intensiva por parte de los médicos de otras especialidades respecto a la suya, los dos grupos consideraron las horas de trabajo, la influencia en la vida personal, el prestigio, el riesgo de mala práctica, la disponibilidad laboral y la sensación de satisfacción del personal como características similares a las de su especialidad. Para el $76 \%$ de los adjuntos y el $70,7 \%$ de los residentes el nivel de estrés fue la única característica que consideraron que era mayor que el que padecían en su especialidad (tabla 5).

Los encuestados tuvieron que escoger y ordenar las características más atractivas de la especialidad por orden de preferencia. En primer lugar quedó el «poder tratar enfermos en situación crítica», característica elegida por el $60 \%$ de los médicos adjuntos y por el 43,9\% de los residentes (tabla 6). En segundo lugar la característica más elegida fue el «poder aplicar conceptos fisiológicos complejos» para el $32,85 \%$ de los adjuntos; sin embargo, para los residentes fue en el $41,5 \%$ «la realización de procedimientos y técnicas», característica esta que fue elegida en tercer lugar por los adjuntos $(28,57 \%)$.

Por último, las características menos atractivas que tenía la especialidad de Medicina Intensiva para los encuestados (tabla 7) fue, en primer lugar, el «estrés del personal» para el $30 \%$ de los adjuntos y para el $26,8 \%$ de los residentes. El segundo lugar lo ocupó el «contacto con cuestiones éticas complejas» $(27,4 \%$ frente al $36,6 \%$ ).

\section{DISCUSIÓN}

De las encuestas distribuidas, tan sólo se recogió algo más de la tercera parte, que correspondía a los

TABLA 2. Respuestas de los profesionales entrevistados a la pregunta: ¿Qué es para usted una Unidad de Cuidados Intensivos?

\begin{tabular}{|c|c|c|}
\hline & $\begin{array}{c}\text { Adjuntos } \\
\mathrm{n}=75\end{array}$ & $\begin{array}{c}\text { Residentes } \\
\mathrm{n}=41\end{array}$ \\
\hline Una sala para enfermos con respirador & $6(8 \%)$ & $3(7,3 \%)$ \\
\hline Una sala donde se controla las 24 horas del día el ritmo del corazón & $6(8 \%)$ & $2(4,8 \%)$ \\
\hline Una sala para enfermos terminales irrecuperables y que se ingresan para evitarles el sufrimiento & 0 & 0 \\
\hline Una sala para enfermos que aunque no estén críticos pudiesen estarlo en cualquier momento & $22(29,3 \%)$ & $10(24,4 \%)$ \\
\hline $\begin{array}{l}\text { Una sala para el tratamiento de pacientes recuperables con una enfermedad que se encuentre } \\
\text { en una fase crítica }\end{array}$ & $74(98,6 \%)$ & $40(97,56 \%)$ \\
\hline $\begin{array}{l}\text { Una sala que pueda tratar enfermos que, aun no estando en fase crítica, supongan una sobrecarga } \\
\text { de trabajo para una planta de hospitalización }\end{array}$ & $2(2,6 \%)$ & $1(2,43 \%)$ \\
\hline
\end{tabular}

Podían elegir entre más de una opción. 


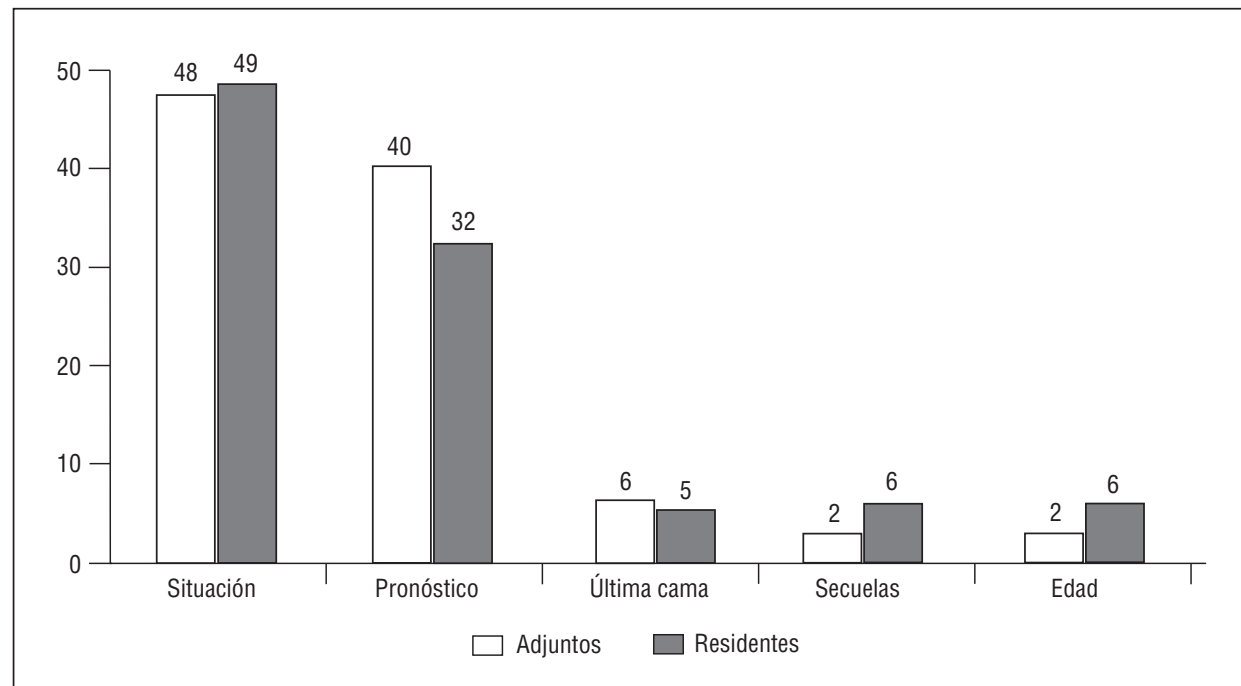

Figura 2. Diferentes motivos de disconformidad ante la negativa de un ingreso en la Unidad de Cuidados Intensivos (\%).

TABLA 3. Causas por las que no solicitó cama en la Unidad de Cuidados Intensivos (UCl)

\begin{tabular}{|l|c|c|}
\hline & $\begin{array}{c}\text { Adjuntos } \\
\mathrm{n}=33\end{array}$ & $\begin{array}{c}\text { Residentes } \\
\mathrm{n}=18\end{array}$ \\
\hline Dar por hecho que no hay camas disponibles en la UCI & $6(18,2 \%)$ & $5(27,7 \%)$ \\
Pensar que se le va a denegar el ingreso en la UCI & $12(36,4 \%)$ & $10(55,5 \%)$ \\
No tener claros los criterios de ingreso en la UCI & $5(15,1 \%)$ & $4(22,2 \%)$ \\
Pensar que se podía tratar de igual forma en planta & $10(30,3 \%)$ & $5(27,7 \%)$ \\
Otras & $3(9,1 \%)$ & 0 \\
\hline
\end{tabular}

Podían elegir entre más de una opción.

servicios que más relación laboral tienen con la UCI. De hecho, la población encuestada poseía amplios conocimientos personales y laborales del Servicio de Medicina Intensiva de su hospital, y un porcentaje importante de ellos había trabajado en alguna UCI durante un periodo medio de casi 4 años. Por todo ello creemos que las conclusiones extraídas de los resultados son de gran valor en cuanto al conocimiento o no de la especialidad y de la UCI; esto no quiere decir que a través de esta relación y su percepción se pueda evaluar de forma eficaz la calidad de la actividad asistencial, ya que la calidad podría ser buena y ser percibida, por diferentes circunstancias, de forma negativa o viceversa.
La experiencia vivida por los familiares de los pacientes ingresados en la UCI ha sido analizada en diferentes estudios desde la perspectiva del profesional, tanto del médico como de la enfermería ${ }^{12-14}$, pero no hemos encontrado ningún trabajo que analice la experiencia vivida por el familiar del paciente ingresado en la UCI cuando este es un profesional médico. Por los resultados de la encuesta, la experiencia vivida por nuestros profesionales, familiares de pacientes ingresados en la UCI en alguna ocasión, parece que es buena, ya que un alto porcentaje lo contestó así.

En una determinada población española, el $48 \%$ no reconocía a los profesionales médicos de los Servicios de Medicina Intensiva como especialistas

TABLA 4. Razones justificadas para no ingresar un paciente

\begin{tabular}{|c|c|c|}
\hline & $\begin{array}{c}\text { Adjuntos } \\
\mathrm{n}=75\end{array}$ & $\begin{array}{c}\text { Residentes } \\
\mathrm{n}=41\end{array}$ \\
\hline La edad & $4(5,8 \%)$ & $6(15,8 \%)$ \\
\hline La situación basal & $47(68,1 \%)$ & $30(78,9 \%)$ \\
\hline El pronóstico a corto plazo & $47(68,1 \%)$ & $20(52,6 \%)$ \\
\hline Las secuelas que le pueden quedar & $4(5,8 \%)$ & $9(15,8 \%)$ \\
\hline Negativa del paciente o de la familia & $53(76,8 \%)$ & $26(68,4 \%)$ \\
\hline La última cama libre en la UCI si el estado del paciente lo permite & $11(15,9 \%)$ & $5(13,1 \%)$ \\
\hline
\end{tabular}

Podían elegir entre más de una opción.

UCI: Unidad de Cuidados Intensivos. 
TABLA 5. Percepción de la especialidad de Medicina Intensiva por los médicos de otras especialidades respecto a la suya

\begin{tabular}{|l|c|c|}
\hline & $\begin{array}{c}\text { Adjuntos } \\
\mathrm{n}=75\end{array}$ & $\begin{array}{c}\text { Residentes } \\
\mathrm{n}=41\end{array}$ \\
\hline Nivel de estrés & $4,12 \pm 0,93$ & $4,07 \pm 0,98$ \\
Horas de trabajo & $3,14 \pm 0,80$ & $3,31 \pm 0,81$ \\
Influencia en la vida personal & $3,16 \pm 0,71$ & $3,60 \pm 0,87$ \\
$\begin{array}{l}\text { Prestigio dentro de la profesión } \\
\quad \text { médica }\end{array}$ & $3,32 \pm 0,83$ & $3,36 \pm 0,95$ \\
Prestigio dentro de la institución & $3,34 \pm 0,90$ & $3,17 \pm 0,91$ \\
Prestigio a nivel social & $3,77 \pm 0,87$ & $3,24 \pm 0,86$ \\
Riesgo de mala práctica & $3,34 \pm 0,96$ & $3,56 \pm 0,98$ \\
Disponibilidad laboral & $3,16 \pm 0,78$ & $2,90 \pm 0,99$ \\
$\begin{array}{l}\text { Sensación de satisfacción } \\
\text { del personal }\end{array}$ & $2,76 \pm 0,78$ & $2,87 \pm 0,86$ \\
\hline
\end{tabular}

Los datos son presentados como medias \pm desviación estándar en respuesta a una escala de valoración de 5 puntos: 1 : mucho menos; 2: algo menos; 3 : similar; 4: algo más; 5 : mucho más.

específicamente de $\mathrm{UCI}^{4}$. En nuestro estudio nos llamó la atención que el 5,3\% de los médicos adjuntos entrevistados aún pensara que los médicos que trabajan en la UCI no son especialistas en Medicina Intensiva y menos de la tercera parte del personal entrevistado creyera que la UCI era para enfermos potencialmente críticos. Por ello creemos que se debe trabajar en los planes estratégicos para mejorar el conocimiento de la especialidad de Medicina Intensiva ${ }^{15}$ tanto entre la población general como entre los propios profesionales sanitarios de otras especialidades. La información a través de los folletos de acogida disponibles para pacientes y familiares, de los que la SEMICYUC ha elaborado un modelo básico que se puede encontrar en su página web, ayuda a difundir todos estos conceptos entre la población general.

$\mathrm{El}$ «modelo español» de Medicina Intensiva, similar en otros países, se caracteriza por considerar que su práctica requiere poseer una serie de conocimientos, habilidades y aptitudes psíquicas y humanas que deben adquirirse bajo un programa de formación específico supervisado y avalado oficialmente por las autoridades sanitarias y académicas, integrado desde el año 1978 en el sistema de Médicos Internos Residentes; además, exige la dedicación a tiempo completo de los pacientes integrados en su ámbito de actuación, pero es compatible con la labor docente e investigadora. En cambio, en Europa y en Estados Unidos la especialidad de Medicina Intensiva no es una especialidad independiente, sino multidisciplinaria, con lo que a partir de especialidades como Anestesia, Medicina Interna, Neumología, Cirugía, etc., y tras un periodo de formación específico, se adquieren las competencias necesarias para ejercer la especialidad $^{16,17}$. En Estados Unidos existen cuatro modelos de asistencia a los pacientes críticos en relación al grado de implicación de los intensivistas en su cuidado; así, en las UCI cerradas el intensivista es el médico responsable del enfermo durante su estancia en la Unidad, mientras que en la UCI abierta el médico responsable sigue siendo el que lo remitió a la UCI, sin ninguna participación del intensivista. Pronovost et al ${ }^{18}$ publicaron el resultado de una revisión sistemática que mostraba que las UCI cerradas se asociaban a una reducción en la mortalidad y en la estancia en UCI y hospitalaria respecto a las UCI abiertas.

A más de la mitad de la población encuestada se le había denegado la solicitud de ingreso de algún paciente, que podría estar o no justificada; el problema es que la mayoría de los médicos quedaron insatisfechos con los argumentos que se les dieron para no ingresar a su paciente. Esto puede justificar el que más del $40 \%$ de los médicos afirmara no haber solicitado cama en la UCI para algún paciente que creía que se podría haber beneficiado de ella, pensando que se le iba a denegar el ingreso. Sería importante insistir en la necesidad de crear un clima de confianza para que el resto de los médicos puedan solicitar la colaboración del intensivista, ya que la UCI debe ser un servicio abierto donde no sólo se ingresan pacientes, sino que también se puede colaborar con el tratamiento del paciente grave que, por diferentes motivos, no ingresa en nuestro Servicio.

También detectamos que un porcentaje no despreciable de la población estudiada no tenía claros los criterios de ingreso en la UCI. Sería interesante en este sentido elaborar guías clínicas de orientación para la actuación a la hora de la admisión de los pacientes crí$\operatorname{ticos}^{19,20}$ y darlos a conocer entre el personal, para lo cual se pueden seguir los criterios generales de priorización de ingreso en los Servicios de Medicina Intensiva que permitan seleccionar a aquellos pacientes que se beneficiarían de ser atendidos en la $\mathrm{UCI}^{3}$.

TABLA 6. Características más atractivas de la especialidad de Medicina Intensiva para los profesionales

\begin{tabular}{|c|c|c|}
\hline & $\begin{array}{c}\text { Adjuntos } \\
\mathrm{n}=70\end{array}$ & $\begin{array}{l}\text { Residentes } \\
\mathrm{n}=41\end{array}$ \\
\hline Estímulo intelectual & $4(5,7 \%)$ & $3(7,3 \%)$ \\
\hline Tratar enfermos en situación crítica & $42(60 \%)$ & $18(43,9 \%)$ \\
\hline Poder aplicar conceptos fisiológicos complejos & $14(20 \%)$ & $10(24,4 \%)$ \\
\hline La realización de procedimientos y técnicas & $5(7,1 \%)$ & $6(14,6 \%)$ \\
\hline El contacto con cuestiones del final de la vida & 0 & 0 \\
\hline La oportunidad de coordinar varios servicios en el tratamiento de un paciente & $4(5,7 \%)$ & $4(9,7 \%)$ \\
\hline El contacto con cuestiones éticas complejas & 0 & 0 \\
\hline Estar de acuerdo con mi personalidad & $1(1,4 \%)$ & 0 \\
\hline Tratar enfermos de forma crónica & 0 & 0 \\
\hline Tener tiempo de ocio durante el trabajo & 0 & 0 \\
\hline Buen ambiente y relax entre el personal & 0 & 0 \\
\hline
\end{tabular}


TABLA 7. Características menos atractivas de la especialidad de Medicina Intensiva para los profesionales

\begin{tabular}{|c|c|c|}
\hline & $\begin{array}{c}\text { Adjuntos } \\
\mathrm{n}=70(\%)\end{array}$ & $\begin{array}{l}\text { Residentes } \\
\mathrm{n}=41(\%)\end{array}$ \\
\hline Falta de estímulo intelectual & 0 & 0 \\
\hline Tratar enfermos en situación crítica & $2(2,8 \%)$ & $1(2,4 \%)$ \\
\hline Tener que aplicar conceptos fisiológicos complejos & $1(1,4 \%)$ & $1(2,4 \%)$ \\
\hline La realización de procedimientos y técnicas que se llevan a cabo & 0 & 0 \\
\hline El contacto con cuestiones del final de la vida & $18(25,7 \%)$ & $10(24,3 \%)$ \\
\hline Faltan oportunidades para coordinar varios servicios en el tratamiento & $3(4,2 \%)$ & $1(2,4 \%)$ \\
\hline El contacto con cuestiones éticas complejas & $7(9,8 \%)$ & $3(7,2 \%)$ \\
\hline El estrés del personal & $21(30 \%)$ & $11(26,8 \%)$ \\
\hline Ser incompatible con mi personalidad & $3(4,2 \%)$ & $2(4,8 \%)$ \\
\hline Tratar enfermos de forma crónica & $2(2,8 \%)$ & $3(7,2 \%)$ \\
\hline Falta de tiempo libre, demanda demasiado tiempo y esfuerzo & $9(12,6 \%)$ & $7(17 \%)$ \\
\hline Mal ambiente entre el personal & $4(5,6 \%)$ & $2(2,8 \%)$ \\
\hline
\end{tabular}

El que las preferencias del paciente sean una de las principales causas para que un paciente no ingrese en la UCI refleja cómo el principio de autonomía y la incorporación de los deseos del enfermo en la toma de decisiones se ha ido implantando en la relación médico-paciente.

Entre los médicos adjuntos y residentes existe la percepción de que la especialidad de Medicina Intensiva, aun dedicándosele las mismas horas de trabajo, tiene un nivel mayor de estrés que la suya, factor responsable del incremento del riesgo de la aparición del síndrome de burnout entre los trabajadores de Cuidados Intensivos ${ }^{21-24}$. Esto lo corroboramos a la hora de preguntarles cuál es la característica más negativa de nuestra especialidad, pues la elegida es el «estrés del personal». Se ha realizado un estudio entre intensivistas de una determinada población española para investigar el nivel de satisfacción profesional y se ha encontrado que son factores internos característicos de la Medicina Intensiva los que condicionan el nivel de satisfacción, pero que este está influido también por factores externos. Se concluye que existe una voluntad real de cambio pero que, quizá, se quiere mejorar la organización de la Medicina Intensiva y de los Servicios de Medicina Intensiva sin perder los privilegios clásicos contrarios a las nuevas tendencias, más orientadas hacia la actividad out-door y, entonces, más orientadas a la obtención de ventaja competitiva ${ }^{25}$.

En conclusión, creemos que la herramienta utilizada puede ser útil para analizar la imagen que damos los profesionales de la Medicina Intensiva de los cuidados ofrecidos dentro del hospital y así poner en marcha los mecanismos de control de calidad pertinentes. En nuestro caso, creemos que deberíamos trabajar el conocimiento entre los profesionales del hospital de lo que es la Medicina Intensiva y los criterios de ingreso, asegurando una adecuada comunicación con el médico que nos solicita algún ingreso; para ello, es importante la participación en sesiones hospitalarias o la difusión de la cartera de servicios a todos los profesionales del hospital. Y sería nuestra obligación no sólo difundir los contenidos y características de nuestra especialidad, sino también mejorar la in- terdisciplinariedad necesaria entre todos los profesionales implicados compartiendo no sólo los criterios de ingreso, sino también la toma de decisiones a lo largo de todo el proceso asistencial.

\section{Declaración de conflicto de intereses}

Los autores han declarado no tener ningún conflicto de intereses.

\section{BIBLIOGRAFÍA}

1. Comisión Nacional de Medicina Intensiva del Consejo Nacional de especialidades Médicas. Programa de formación para médicos especialistas en Medicina Intensiva. Med Intensiva. 1993; 17:293-300

2. Instituto Nacional de la Salud. Subdirección General de Coordinación Administrativa. Guía para la coordinación, evaluación y gestión de los servicios de Medicina Intensiva. Madrid; 1997.

3. Guidelines for intensive care unit admission, discharge, and triage. Task Force of the American College of Critical Care Medicine, Society of Critical Care Medicine. Crit Care Med. 1999; 27:633-8.

4. García-Labattut A, Tena F, Díaz J, Pajares R, Sandoval J, González J, et al, por la Sociedad Castellano-Leonesa de Medicina Intensiva, Crítica y de Unidades Coronarias. Nivel de conocimiento sobre las Unidades de Cuidados Intensivos (UCI) y los médicos intensivistas entre los ciudadanos de Castilla y León. Med Intensiva. 2006;30:45-51.

5. Cohn SM, Price MA, Stewart RM, Pruitt BA Jr, Dent DL. Perceptions and attitudes of critical care training and careers among United States surgical residents: who wants to be a surgical intensivist? Am Surg. 2007;73:433-7.

6. Lorin S, Heffner J, Carson S. Attitudes and perceptions of internal medicine residents regarding pulmonary and critical care subspecialty training. Chest. 2005;127:630-6.

7. Dodek PM, Heyland DK, Rocker GM, Cook DJ. Translating family satisfaction data into quality improvement. Crit Care Med. 2004;32:1922-7.

8. Curtis JR, Cook DJ, Wall RJ, Angus DC, Bion J, Kacmarek $\mathrm{R}$, et al. Intensive care unit quality improvement: A "how to" guide for the interdisciplinary team. Crit Care Med. 2006;34:211-8.

9. Dowling J, Vender J, Guilianelli S, Wang B. A model of Family-Centered Care and Satisfaction Predictors: the Critical Care Family Assistance Program. Chest. 2005;128:81S-92S.

10. Gil J, Rodríguez J, Díaz-Alersi R, Martínez P, Huertos M, Ruiz M. La comunicación con el usuario y sus familiares en una Unidad de Medicina Intensiva. Interés de la encuesta post-alta. Med Intensiva. 1994;18:55-60. 
11. Sociedad Española de Medicina Intensiva Crítica y Unidades Coronarias. Indicadores de calidad en el enfermo crítico. 1. ${ }^{a}$ ed. Madrid; 2005. Disponible en: http://www.calidad.semicyuc.org

12. Santana Cabrera L, Sánchez Palacios M, Hernández Medina E, García Martul M, Eugenio Robaina P, Villanueva Ortiz A. Necesidades de los familiares de pacientes de cuidados intensivos: percepción de los familiares y del profesional. Med Intensiva. 2007;31:273-80.

13. Bijttebier P, Vanoost S, Delva D, Ferdinande P, Frans E. Needs of relatives of critical care patients: perceptions of relatives, physicians and nurses. Intensive Care Med. 2001;27:160-5.

14. Myhren H, Ekeberg O, Langen I, Stokland O. Emotional strain, communication, and satisfaction of family members in the intensive care unit compared with expectations of the medical staff: experiences from a Norwegian University Hospital. Intensive Care Med. 2004;30:1791-8.

15. Martín MC, Ruiz J, Blanch L, Díaz E. Plan estratégico de la Sociedad Catalana de Medicina Intensiva y Crítica (S.O.C.M.I.C.) Med Intensiva. 2003;27:32-41.

16. Ruiz Moreno J, Martín Delgado MC. Ciento cincuenta años de atención al enfermo crítico. JANO. 2006;1614:47-52.

17. De Lange S, Van Aken H, Burchardi H. European Society of Intensive Care Medicine statement: Intensive care medicine in Europe - structure, organisation and training guidelines of the Multidisciplinary Joint Committee of Intensive Care Medicine
(MJCICM) of the European Union of Medical Specialists (UEMS). Intensive Care Med. 2002;28:1505-11.

18. Pronovost PJ, Angus DC, Dorman T, Robinson KA, Dremsizov TT, Young TL. Physician staffing patterns and clinical outcomes in critically ill patients: a systematic review. JAMA 2002;288:2151-62.

19. Martin DK, Singer PA, Bernstein M. Access to intensive care unit beds for neurosurgery patients: a qualitative case study. J Neurol Neurosurg Psychiatry. 2003;74:1299-303.

20. Sinuff T, Kahnamoui K, Cook DJ, Luce JM, Levy MM. Values Ethics and Rationing in Critical Care Task Force. Rationing critical care beds: a systematic review. Crit Care Med. 2004;32: 1588-97.

21. Embriaco N, Papazian L, Kentish-Barnes N, Pochard F, Azoulay E. Burnout syndrome among critical care healthcare workers. Curr Opin Crit Care. 2007;13:482-8.

22. Raggio B, Malacarne P. Burnout in intensive care unit. Minerva Anestesiol. 2007;73:195-200

23. Guntupalli KK, Fromm RE Jr. Burnout in the internist-intensivist. Intensive Care Med. 1996;22:625-30.

24. Buhler KE, Land T. Burnout and personality in intensive care: an empirical study. Hosp Top. 2003 Fall;81:5-12.

25. Ruiz J, Martín MC, Morillas J, Ricart P, Blanch Ll, Armengol S, et al. Análisis del nivel de satisfacción profesional y de la visión de futuro de la medicina intensiva como especialidad. Med Intensiva. 2002;26:51-60. 